\title{
THE MOMENTS OF MINKOWSKI QUESTION MARK FUNCTION: THE DYADIC PERIOD FUNCTION
}

\author{
GIEDRIUS ALKAUSKAS \\ The Department of Mathematics and Informatics, Vilnius University, Naugarduko 24, Vilnius, Lithuania and \\ School of Mathematical Sciences, University of Nottingham, University Park, Nottingham NG7 2RD, UK \\ e-mail: giedrius.alkauskas@gmail.com
}

(Received 28 October 2008; accepted 22 May 2009)

\begin{abstract}
The Minkowski question mark function?(x) arises as a real distribution of rationals in the Farey tree. We examine the generating function of moments of $?(x)$. It appears that the generating function is a direct dyadic analogue of period functions for Maass wave forms and it is defined in the cut plane $\mathbb{C} \backslash(1, \infty)$. The exponential generating function satisfies an integral equation with kernel being the Bessel function. The solution of this integral equation leads to the definition of dyadic eigenfunctions, arising from a certain Hilbert-Schmidt operator. Finally, we describe $p$-adic distribution of rationals in the Stern-Brocot tree. Surprisingly, the Eisenstein series $G_{2}(z)$ does manifest in both real and $p$-adic cases.

2000 Mathematics Subject Classification. Primary - 11A55, 26A30, 11F03; Secondary $-33 \mathrm{C} 10$.
\end{abstract}

1. Introduction. This paper is the first in the series of four papers (others being [1-3]) which are devoted to the study of moments and integral transforms of the Minkowski question mark function (unfortunately, it appears in print the last).

The function ?( $x$ ) ('the question mark function') was introduced by Minkowski in 1904 [29] as an example of continuous and monotone function ? : $[0,1] \rightarrow[0,1]$, which maps rationals to dyadic rationals, and quadratic irrationals to non-dyadic rationals. It is though more convenient to work with the function $F(x):=?\left(\frac{x}{x+1}\right), x \in[0, \infty) \cup\{\infty\}$. Thus, for non-negative real $x$ it is defined by the expression

$$
F\left(\left[a_{0}, a_{1}, a_{2}, a_{3}, \ldots\right]\right)=1-2^{-a_{0}}+2^{-\left(a_{0}+a_{1}\right)}-2^{-\left(a_{0}+a_{1}+a_{2}\right)}+\cdots,
$$

where $x=\left[a_{0}, a_{1}, a_{2}, a_{3}, \ldots\right]$ stands for the representation of $x$ by a (regular) continued fraction [20]. Hence, according to our convention, ? $(x)=2 F(x)$ for $x \in[0,1]$. For rational $x$ the series terminates at the last non-zero element $a_{n}$ of the continued fraction.

1.1. Short literature overview. The Minkowski question mark function was investigated by many authors. In this subsection we give an overview of available literature.

Denjoy [9] gave an explicit expression for $F(x)$ in terms of a continued fraction expansion, that is, formula (1). He also showed that ?(x) is purely singular: the derivative, in terms of the Lebesgue measure, vanishes almost everywhere. Salem [38] proved that ?(x) satisfies the Lipschitz condition of order $\frac{\log 2}{2 \log \gamma}$, where $\gamma=\frac{1+\sqrt{5}}{2}$, and this is in fact the best possible exponent for the Lipschitz condition. The 


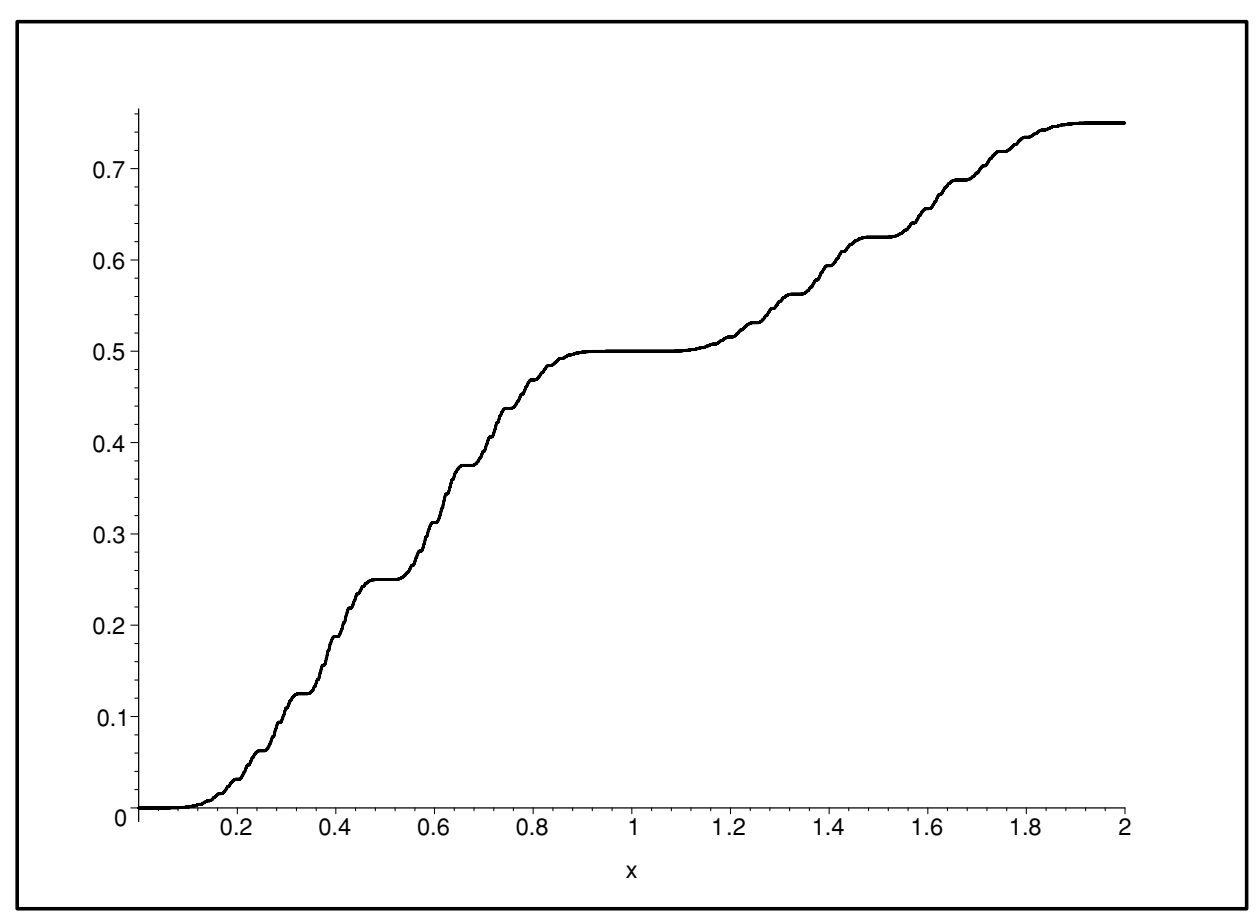

Figure 1. The Minkowski question mark function $F(x), x \in[0,2]$.

Fourier-Stieltjes coefficients of ?(x), defined as $\int_{0}^{1} e^{2 \pi i n x} \mathrm{~d} ?(x)$, where also investigated in the same paper (these coefficients also appeared in [5]; see also [36]). The author, as an application of Wiener's theorem about Fourier series, gives average results on these coefficients without giving an answer to yet unsolved problem whether these coefficients vanish, as $n \rightarrow \infty$ (it is worth noting that in [2] analogous Fourier coefficients are introduced and examined). Kinney [21] proved that the Hausdorff dimension of growth points of ? $(x)$ (denote this set by $\mathcal{A})$ is equal to $\alpha=\frac{1}{2}\left(\int_{0}^{1} \log _{2}(1+x) \mathrm{d} ?(x)\right)^{-1}$. Numerical estimates for this constant were obtained in [23] and [42]; based on a three term functional equation, we are able to calculate the Kinney's constant to a high precision (in the appendix of [3] we calculate 35 exact digits; note that some digits presented in [37] are incorrect). Also, if $x_{0} \in \mathcal{A}, ?(x)$ at a point $x_{0}$ satisfies the Lipschitz condition with exponent $\alpha$. The function ? $(x)$ is mentioned in [8] in connection with a 'box' function. In [24] Lagarias and Tresser introduce the so called $\mathbb{Q}$-tree: an extension of the Farey tree which contains all (positive and negative) rationals. Tichy and Uitz [42] extended Kinney's approach (mainly, calculation of a Hausdorff dimension) to a parametrised class of singular functions related to ?(x). C. Bower, unpublished note, 1999 considers the solution of the equation $?(x)=x$, different from $x=0, \frac{1}{2}$ or 1 . There are two of them (symmetric with respect to $x=\frac{1}{2}$ ), the first one is given by $x=0.42037233_{+}$ (S. R. Finch, unpublished note, 2008). Apparently, no closed form formula exists for it. In [10] Dilcher and Stolarsky introduced what they call Stern polynomials. The construction is analogous to similar constructions given in $[\mathbf{3}, \mathbf{1 3}]$. Nevertheless, in [10] all polynomials have coefficients 0 and 1 , and their structure is compatible with regular continued fraction algorithm. In [11] Dushistova and Moshchevitin find conditions in order $?^{\prime}(x)=0$ and $?^{\prime}(x)=\infty$ to hold (for certain fixed positive real $x$ ) 
in terms of $\limsup \sup _{t \rightarrow \infty} \frac{a_{0}+a_{1}+\cdots+a_{t}}{t}$ and $\liminf _{t \rightarrow \infty} \frac{a_{0}+a_{1}+\cdots+a_{t}}{t}$ respectively, where $x=\left[a_{0}, a_{1}, a_{2}, \ldots\right]$ is represented by a continued fraction. The nature of singularity of ? $(x)$ was clarified by Viader, Paradís and Bibiloni [34]. In particular, the existence of the derivative ?' $(x)$ in $\mathbb{R}$ for fixed $x$ forces it to vanish. Some other properties of ?(x) are demonstrated in [33]. In [19] Kesseböhmer and Stratmann studied various fractal geometric aspects of the Minkowski question mark function $F(x)$. They showed that the unit interval can be written as the union of three sets: $\Lambda_{0}:=\left\{x: F^{\prime}(x)=\right.$ $0\}, \Lambda_{\infty}:=\left\{x: F^{\prime}(x)=\infty\right\}$, and $\Lambda_{\sim}:=\left\{x: F^{\prime}(x)\right.$ does not exist and $\left.F^{\prime}(x) \neq \infty\right\}$. Their main result is that the Hausdorff dimensions of these sets are related in the following way: $\operatorname{dim}_{H}\left(v_{F}\right)<\operatorname{dim}_{H}\left(\Lambda_{\sim}\right)=\operatorname{dim}_{H}\left(\Lambda_{\infty}\right)=\operatorname{dim}_{H}\left(\mathscr{L}\left(h_{\text {top }}\right)\right)<\operatorname{dim}_{H}\left(\Lambda_{0}\right)=1$. Here $\mathscr{L}\left(h_{\text {top }}\right)$ refers to the level set of the Stern-Brocot multifractal decomposition at the topological entropy $h_{\text {top }}=\log 2$ of the Farey map $Q$, and $\operatorname{dim}_{H}\left(v_{F}\right)$ denotes the Hausdorff dimension of the measure of maximal entropy of the dynamical system associated with $Q$. The notions and technique were developed earlier by authors in [18]. The paper (J. C. Lagarias, unpublished manuscript, 1991), deals with the interrelations among the additive continued fraction algorithm, the Farey tree, the Farey shift and the Minkowski question mark function. The motivation for the work [32] is a fact that the function ? $(x)$ can be characterised as the unique homeomorphism of the real unit interval that conjugates the Farey map with the tent map. In [32] Panti constructs an $n$-dimensional analogue of the Minkowski function as the only homeomorphism of an $n$-simplex that conjugates the piecewise-fractional map associated to the Mönkemeyer continued fraction algorithm with an appropriate tent map. In [5] Bonanno and Isola introduce a class of one-dimensional maps which can be used to generate the binary trees in different ways, and study their ergodic properties. This leads to studying some random processes (Markov chains and martingales) arising in a natural way in this context. In the course of the paper, the authors also introduce a function $\rho(x)=?\left(\frac{x}{x+1}\right)$, which is, of course, exactly $F(x)$. Okamoto and Wunsch [31] construct yet another generalisation of ? $(x)$, though their main concern is to introduce a new family of purely singular functions. Meanwhile, the paper by Grabner, Kirschenhofer and Tichy [15], out of all papers in the bibliography list, is the closest in spirit to the current article. In order to derive precise error bounds for the so called Garcia entropy of a certain measure, the authors consider the moments of the continuous and singular function $F_{2}\left(\left[a_{1}, a_{2}, \ldots\right]\right)=\sum_{n=1}^{\infty}(-1)^{n-1} 3^{-\left(a_{1}+\cdots+a_{n}-1\right)}\left(q_{n}+q_{n-1}\right)$, where $q_{\star}$ stand for a corresponding denominator of the convergent to $\left[a_{1}, a_{2}, \ldots\right]$. Lamberger [25] showed that $F(x)$ and $F_{2}(x)$ are the first two members of a family (indexed by natural numbers) of mutually singular measures, derived from the subtractive Euclidean algorithm. The latter two papers are very interesting and promising, and the author of this article does intend to generalise the results about $F(x)$ to the whole family $F_{j}(x), j \in \mathbb{N}$. Other references include $[4,14,16,35,37]$.

1.2. Stern-Brocot tree. Recently, Calkin and Wilf [6] (re-)defined a binary tree which is generated by the iteration

$$
\frac{a}{b} \mapsto \frac{a}{a+b}, \frac{a+b}{b},
$$

starting from the root $\frac{1}{1}$ (this tree is a permutation of the well-known Stern-Brocot tree). Elementary considerations show that this tree contains any positive rational number once and only once, each represented in lowest terms [6]. First four iterations 
lead to

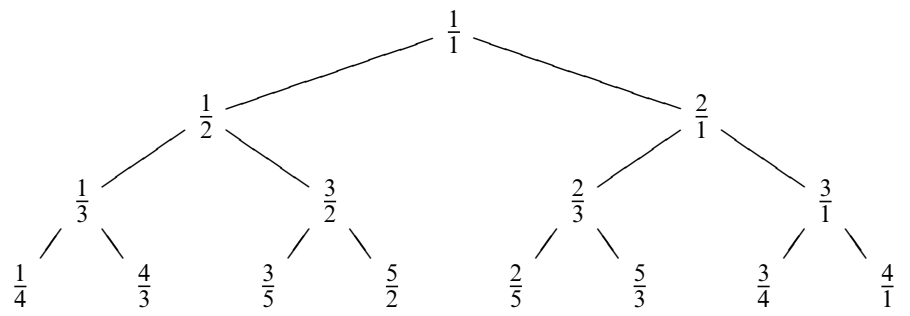

Thus, the $n$th generation consists of $2^{n-1}$ positive rationals $x_{n}^{(i)}, 1 \leq i \leq 2^{n-1}$. We denote this tree by $\mathcal{T}$, and its $n$th generation by $\mathcal{T}^{(n)}$. The limitation of this tree to the interval $[0,1]$ is the well-known Farey tree (albeit with different order). Reading the tree line by line, this enumeration of $\mathbb{Q}_{+}$starts with

$$
\frac{1}{1}, \frac{1}{2}, \frac{2}{1}, \frac{1}{3}, \frac{3}{2}, \frac{2}{3}, \frac{3}{1}, \frac{1}{4}, \frac{4}{3}, \frac{3}{5}, \frac{5}{2}, \frac{2}{5}, \frac{5}{3}, \frac{3}{4}, \frac{4}{1}, \ldots
$$

This sequence was already investigated by Stern [41], where one encounters the definition of the Stern-Brocot tree. The sequence satisfies the remarkable iteration discovered by Newman [30]:

$$
x_{1}=1, \quad x_{n+1}=1 /\left(2\left[x_{n}\right]+1-x_{n}\right),
$$

thus giving an example of a simple recurrence which produces all positive rationals, and answering affirmatively to a question by D. E. Knuth. The $n$th generation of $\mathcal{T}$ consists of exactly those rational numbers whose elements of the continued fraction sum up to $n$; this observation is due to Stern [41]. Indeed, this can be easily inherited directly from the definition. First, if rational number $\frac{a}{b}$ is represented as a continued fraction $\left[a_{0}, a_{1}, \ldots, a_{r}\right]$, then the map $\frac{a}{b} \rightarrow \frac{a+b}{b}$ maps $\frac{a}{b}$ to $\left[a_{0}+1, a_{1} \ldots, a_{r}\right]$. Second, the map $\frac{a}{b} \rightarrow \frac{a}{a+b}$ maps $\frac{a}{b}$ to $\left[0, a_{1}+1, \ldots, a_{r}\right]$ in case $\frac{a}{b}<1$, and to $\left[0,1, a_{0}, a_{1}, \ldots, a_{r}\right]$ in case $\frac{a}{b}>1$. This simple fact is of utmost importance in our work: though it is not used in explicit form, this highly motivates the investigations of moments $M_{L}$ and $m_{L}$, given by (5). The sequence of numerators of the Calkin-Wilf tree

$$
0,1,1,2,1,3,2,3,1,4,3,5,2,5,3,4,1, \ldots
$$

is called the Stern diatomic sequence $[\mathbf{2 9}, \mathbf{4 4}]$ and it satisfies the recurrence relations

$$
s(0)=0, \quad s(1)=1, \quad s(2 n)=s(n), \quad s(2 n+1)=s(n)+s(n+1) .
$$

In the next section we will show that each generation of the Calkin-Wilf tree possesses a distribution function $F_{n}(x)$, and $F_{n}(x)$ converges uniformly to $F(x)$. This is by far not a new fact. Nevertheless, we include the short proof of it for the sake of selfcontainedness. The function $F(x)$, as a distribution function, is uniquely determined by the functional equation (3). This implies the explicit expression (1) and the symmetry property $F(x)+F(1 / x)=1$. The mean value of $F(x)$ was investigated by several authors (J. Steuding, personal communication, 2006 and [48]) and was proved to be $3 / 2$. We will obtain this result using quite a different method. 
1.3. Motivation and description of results. The aim of this paper is to give a different treatment of Minkowski's ?(x). All papers so far were concerned with $F(x)$ as the function per se. Nevertheless, it appears that there exist several natural integral transforms of $F(x)$, which are analytic functions and which encode certain substantial (in fact, all) information about the question mark function. Each of these transforms is characterised by regularity properties and a functional equation. Lastly and most importantly, let us point out that, surprisingly, there are striking similarities and analogies between the results proved here as well as in [2, 3] with Lewis-Zagier's [28] results on period functions for Maass wave forms. That work is an expanded and clarified exposition of an earlier paper by Lewis [27]. The concise exposition of these objects, their properties and relations to Selberg zeta function can be found in [46]. The reader is strongly urged to compare results in this work with those in [28]. Let, for example, $u(z)$ be a Maass wave form for $\mathrm{PSL}_{2}(\mathbb{Z})$ with spectral parameter $s$. The similarity arises due to the fact that the limit value of $u(z)$ on the real line, given by $u(x+i y) \sim y^{1-s} U(x)+y^{s} U(x)$ as $y \rightarrow 0+$, satisfies (formal) functional equations $U(x+1)=U(x)$ and $|x|^{2 s-2} U\left(-\frac{1}{x}\right)=U(x)$. Thus, these are completely analogous to the functional equations for $F(x)$, save the fact that $U(x)$ is only a formal function - it is a distribution (e.g. a continuous functional in properly defined space of functions). Thus, our objects $G(z), \mathfrak{m}(t)$ and $M(t)$ are analogues of objects $\psi(z), g(w)$ and $\phi(w)$ respectively (see Section 2 of this work and [28]). In [2] it is shown that in fact $L$ functions attached to Maass wave forms also do have an analogue in context of the Minkowski question mark function.

This work is organised as follows. In Section 2 we demonstrate some elementary properties of the distribution function $F(x)$. Since the existence of all moments is guaranteed by the exponential decay of the tail, our main object is the generating function of moments, denoted by $G(z)$. In Section 3 we prove two functional equations for $G(z)$. In Section 4 we demonstrate the uniqueness of solution of this functional equation (subject to regularity conditions). Surprisingly, the Eisenstein series $G_{2}(z)$ appears on the stage. In Section 5 we prove the integral equation for the exponential generating function. In Section 6 a new class of functions emerging from eigenfunctions of a Hilbert-Schmidt operator (we call them 'dyadic eigenfunctions') is introduced. These are dyadic analogues of functions discovered by Wirsing [44] in connection with the Gauss-Kuzmin-Lévy problem. In Section 7 we describe the $p$-adic distribution of rationals in the Calkin-Wilf tree. In the final section, some concluding remarks are presented.

2. Some properties of the distribution. The following proposition was proved by many authors in various forms, concerning (very related) Stern-Brocot, Farey or Calkin-Wilf trees, and this seems to be a well-known fact about a distribution of rationals in these trees. For the sake of completeness we present a short proof, since the functional equations for $G(z)$ and $\mathfrak{m}(t)$ (see Sections 3 and 5 ) heavily depend on the functional equation for $F(x)$ and are in fact reformulations of these in different terms.

Proposition 1. Let $F_{n}(x)$ denote the distribution function of the nth generation, i.e.,

$$
F_{n}(x)=2^{1-n} \#\left\{j: x_{j}^{(n)} \leq x\right\} .
$$

Then uniformly $F_{n}(x) \rightarrow F(x)$. Thus, $F(0)=0, F(\infty)=1$. Moreover, $F(x)$ is continuous, monotone and singular, i.e. $F^{\prime}(x)=0$ almost everywhere. 
Proof. Let $x \geq 1$. One half of the fractions in the $n+1$-st generation do not exceed 1 , and hence also do not exceed $x$. Further,

$$
\frac{a+b}{b} \leq x \quad \Longleftrightarrow \quad \frac{a}{b} \leq x-1
$$

Hence,

$$
2 F_{n+1}(x)=F_{n}(x-1)+1, \quad n \geq 1 .
$$

Now assume $0<x<1$. Then

$$
\frac{a}{a+b} \leq x \quad \Longleftrightarrow \quad \frac{a}{b} \leq \frac{x}{1-x} .
$$

Therefore,

$$
2 F_{n+1}(x)=F_{n}\left(\frac{x}{1-x}\right) .
$$

The distribution function $F$, defined in the formulation of the Proposition 1, satisfies the functional equation

$$
2 F(x)= \begin{cases}F(x-1)+1 & \text { if } \quad x \geq 1 \\ F\left(\frac{x}{1-x}\right) & \text { if } \quad 0<x<1\end{cases}
$$

For instance, the second identity is equivalent to $2 F\left(\frac{t}{t+1}\right)=F(t)$ for all positive $t$. If $t=\left[b_{0}, b_{1}, \ldots\right]$, then $\frac{t}{t+1}=\left[0,1, b_{0}, b_{1}, \ldots\right]$ for $t \geq 1$, and $\frac{t}{t+1}=\left[0, b_{1}+1, b_{2}, \ldots\right]$ for $t<1$, and the statement follows immediately.

Now define $\delta_{n}(x)=F(x)-F_{n}(x)$. In order to prove the uniform convergence $F_{n} \rightarrow$ $F$, it is sufficient to show that

$$
\sup _{x \geq 0}\left|\delta_{n}(x)\right| \leq 2^{-n}
$$

It is easy to see that the assertion is true for $n=1$. Now suppose the estimate is true for $n$. In view of the functional equation for both $F_{n}(x)$ and $F(x)$, we have

$$
2 \delta_{n+1}(x)=\delta_{n}\left(\frac{x}{1-x}\right)
$$

for $0<x<1$, which gives $\sup _{0 \leq x<1}\left|\delta_{n+1}(x)\right| \leq 2^{-n-1}$. Moreover, we have

$$
2 \delta_{n+1}(x)=\delta_{n}(x-1)
$$

for $x \geq 1$, which yields the same bound for $\delta_{n}(x)$ in the range $x \geq 1$. This proves (4). As it was noted, the singularity of $F(x)$ was proved in [9] and it follows from Khinchin's results on metric properties of continued fractions.

Since $F(x)$ has a tail of exponential decay $\left(1-F(x)=O\left(2^{-x}\right)\right.$, as it is clear from (1)), all moments do exist. Let

$$
M_{L}=\int_{0}^{\infty} x^{L} \mathrm{~d} F(x), \quad m_{L}=\int_{0}^{\infty}\left(\frac{x}{x+1}\right)^{L} \mathrm{~d} F(x)=2 \int_{0}^{1} x^{L} \mathrm{~d} F(x)=\int_{0}^{1} x^{L} \mathrm{~d} ?(x) .
$$


Therefore, $M_{L}$ and $m_{L}$ can also be defined as

$$
M_{L}=\lim _{n \rightarrow \infty} 2^{1-n} \sum_{a_{0}+a_{1}+\cdots+a_{s}=n}\left[a_{0}, a_{1}, \ldots, a_{s}\right]^{L}, m_{L}=\lim _{n \rightarrow \infty} 2^{2-n} \sum_{a_{1}+\cdots+a_{s}=n}\left[0, a_{1}, \ldots, a_{s}\right]^{L},
$$

where the summation takes place over all rationals, whose elements of the continued fraction sum up to $n$. These expressions highly motivate our investigation of moments. Though the authors in [15] considered the moments of $F_{2}(x)$ (see the introduction), it is surprising that the moments of Minkowski question mark function itself were never investigated. Numerically, one has

$$
\begin{aligned}
M_{1} & =1.5, \quad M_{2}=4.290926, \quad M_{3}=18.556, \quad M_{4}=107.03 ; \\
m_{1} & =0.5, \quad m_{2}=0.290926, \quad m_{3}=0.186389, \quad m_{4}=0.126992 .
\end{aligned}
$$

We will see that the generating function of $m_{L}$ possesses certain fascinating properties. Let $\omega(x)$ be a continuous function of at most polynomial growth: $\omega(x)=O\left(x^{T}\right), x \rightarrow$ $\infty$. The functional equation (3) gives $F(x+n)=1-2^{-n}+2^{-n} F(x), x \geq 0$. Hence

$$
\begin{aligned}
& \int_{0}^{\infty} \omega(x) \mathrm{d} F(x)=\sum_{n=0}^{\infty} \int_{0}^{1} \omega(x+n) \mathrm{d} F(x+n) \\
& \quad=\int_{0}^{1} \sum_{n=0}^{\infty} \frac{\omega(x+n)}{2^{n}} \mathrm{~d} F(x) \stackrel{x \rightarrow \frac{t}{t+1}}{=} \sum_{n=0}^{\infty} \int_{0}^{\infty} \frac{\omega\left(\frac{t}{t+1}+n\right)}{2^{n+1}} \mathrm{~d} F(t) .
\end{aligned}
$$

Since, as noted above, $F(x)$ has a tail of exponential decay, this integral does exist (all changes of order of summation and integration are easily justifiable minding the condition on $\omega(x))$. Let $\omega(x)=x^{L}, L \in \mathbb{N}_{0}$. Then, if we denote $B_{s}=\sum_{n=0}^{\infty} \frac{n^{s}}{2^{n+1}}$, we have

$$
\int_{0}^{\infty} x^{L} \mathrm{~d} F(x)=\int_{0}^{\infty} \sum_{i=0}^{L}\left(\frac{x}{x+1}\right)^{i}\left(\begin{array}{c}
L \\
i
\end{array}\right) B_{L-i} \mathrm{~d} F(x) .
$$

Whence the relation

$$
M_{L}=\sum_{i=0}^{L} m_{i}\left(\begin{array}{c}
L \\
i
\end{array}\right) B_{L-i}, \quad L \geq 0
$$

The exponential generating function of $B_{L}$ is

$$
B(t)=\sum_{L=0}^{\infty} \frac{B_{L}}{L !} t^{L}=\sum_{L=0}^{\infty} \sum_{n=0}^{\infty} \frac{n^{L} t^{L}}{2^{n+1} L !}=\sum_{n=0}^{\infty} \frac{e^{n t}}{2^{n+1}}=\frac{1}{2-e^{t}}
$$

Denote by $M(t)$ and $\mathfrak{m}(t)$ the corresponding exponential generating functions of the coefficients $M_{L}$ and $m_{L}$, respectively. Accordingly,

$$
M(t)=\int_{0}^{\infty} e^{x t} \mathrm{~d} F(x), \quad \mathfrak{m}(t)=\int_{0}^{\infty} \exp \left(\frac{x t}{x+1}\right) \mathrm{d} F(x)=2 \int_{0}^{1} e^{x t} \mathrm{~d} F(x) .
$$


The relation (6) in terms of $M(t)$ and $\mathfrak{m}(t)$ reads as

$$
M(t)=\sum_{L=0}^{\infty} \frac{M_{L}}{L !} t^{L}=\frac{1}{2-e^{t}} \sum_{L=0}^{\infty} \frac{m_{L}}{L !} t^{L}=\frac{1}{2-e^{t}} \mathfrak{m}(t) .
$$

We see that the function $\mathfrak{m}(t)$ is entire and $M(t)$ has a positive radius of convergence. The last identity implies the asymptotic formula for $M_{L}$.

Proposition 2. For $L \in \mathbb{N}_{0}$,

$$
\begin{aligned}
M_{L} & =\frac{\mathfrak{m}(\log 2)}{2 \log 2}\left(\frac{1}{\log 2}\right)^{L} L !+O_{\varepsilon}\left(\left(4 \pi^{2}+\left(\log ^{2} 2\right)^{1 / 2}-\varepsilon\right)^{-L}\right) L ! \\
& =\left(\frac{\mathfrak{m}(\log 2)}{2 \log 2}\left(\frac{1}{\log 2}\right)^{L}+O\left(6.3^{-L}\right)\right) L !
\end{aligned}
$$

Proof. By Cauchy's formula, for any sufficiently small $r$,

$$
M_{L}=\frac{L !}{2 \pi i} \int_{|z|=r} \frac{M(z)}{z^{L+1}} \mathrm{~d} z
$$

Changing the path of integration, we get by the calculus of residues

$$
M_{L}=-\operatorname{Res}_{z=\log 2}\left(\frac{m(z)}{\left(2-e^{z}\right) z^{L+1}}\right)-\frac{L !}{2 \pi i} \int_{|z|=R} \frac{m(z)}{2-e^{z}} \frac{\mathrm{d} z}{z^{L+1}},
$$

where $R$ satisfies $\log 2<R<|\log 2+2 \pi i|$ (which means that there is exactly one simple pole of the integrand located in the interior of the circle $|z|=R$ ). It is easily seen that the residue coincides with the main term in the formula of the proposition; the error term follows from estimating the integral.

Also, (7) gives the inverse to linear equations (6):

$$
m_{L}=M_{L}-\sum_{s=0}^{L-1} M_{s}\left(\begin{array}{c}
L \\
s
\end{array}\right), \quad L \geq 0
$$

Since $B(t)\left(2-e^{t}\right)=1$, the coefficients $B_{L}$ can be calculated recursively: $B_{L}=$ $\sum_{s=0}^{L-1}\left(\begin{array}{l}L \\ s\end{array}\right) B_{s}$. Thus, $B_{0}=1, B_{1}=1, B_{2}=3, B_{3}=13, B_{4}=75, B_{5}=541$. This sequence has number $A 000670$ in [40], and traces its history back from Cayley.

In the future, we will consider integrals which involve $\mathfrak{m}(t)$, and hence we need the evaluation of this function for negative $t$.

Lemma 1. Let $C=e^{-\sqrt{\log 2}}=0.4349_{+}$. Then $C^{2 \sqrt{t}} \ll \mathfrak{m}(-t) \ll C^{\sqrt{t}}$ as $t \rightarrow \infty$.

Proof. In fact, $\mathfrak{m}(-t)=\int_{0}^{\infty} \exp \left(-\frac{x t}{x+1}\right) \mathrm{d} F(x)$. Hence, $\mathfrak{m}(t)$ is positive for $t \in \mathbb{R}$. Let $0<M<1$. Since $1-F(x) \asymp 2^{-x}$ as $x \rightarrow \infty$, and $F(x)+F(1 / x)=1$,

$$
\mathfrak{m}(-t)=\left(\int_{0}^{M}+\int_{M}^{\infty}\right) \exp \left(-\frac{x t}{x+1}\right) \mathrm{d} F(x) \ll 2^{-1 / M}+\exp \left(-\frac{M t}{M+1}\right) .
$$


This is valid for every $M<1$ and a universal constant. A choice $M=\frac{\sqrt{\log 2}}{\sqrt{t}}$ gives the desired upper bound. To obtain the lower bound, note that

$$
\mathfrak{m}(-t)>\int_{0}^{M} \exp \left(-\frac{x t}{x+1}\right) \mathrm{d} F(x) \gg 2^{-1 / M} \cdot \exp \left(-\frac{M t}{M+1}\right) .
$$

The same choice for $M$ establishes the lower bound. Naturally, similar evaluation holds for the derivative, since $\mathfrak{m}^{\prime}(-t)=\int_{0}^{\infty} \frac{x}{x+1} \exp \left(-\frac{x t}{x+1}\right) \mathrm{d} F(x)$.

We will prove one property of the function $\mathfrak{m}(t)$ which represents the symmetry of $F$ given by $F(x)+F(1 / x)=1$.

Proposition 3. We have: $\mathfrak{m}(t)=e^{t} \mathfrak{m}(-t)$.

Proof. In fact,

$$
\begin{aligned}
\mathfrak{m}(t) & =\int_{0}^{\infty} \exp \left(\frac{x t}{x+1}\right) \mathrm{d} F(x)=-\int_{0}^{\infty} \exp \left(\frac{t / x}{1 / x+1}\right) \mathrm{d} F(1 / x) \\
& =\int_{0}^{\infty} \exp \left(\frac{t}{x+1}\right) \mathrm{d} F(x)=e^{t} \int_{0}^{\infty} \exp \left(-\frac{x t}{x+1}\right) \mathrm{d} F(x)=\mathfrak{m}(-t) e^{t} .
\end{aligned}
$$

Whence the relations

$$
m_{L}=\sum_{s=0}^{L}\left(\begin{array}{l}
L \\
s
\end{array}\right)(-1)^{s} m_{s}, \quad L \geq 0 .
$$

Thus, $m_{1}=m_{0}-m_{1}$, which gives $m_{1}=1 / 2$, and this implies $M_{1}=3 / 2$. Also, $2 m_{3}=$ $-1 / 2+3 m_{2}$. These linear relations are further investigated in [2].

3. The dyadic period function $G(z)$. We introduce the generating power function of moments

$$
\mathcal{M}(z)=\sum_{L=0}^{\infty} m_{L} z^{L}
$$

A priori, this series converges in the unit circle. Recall that $\int_{0}^{\infty} x^{n} e^{-x} \mathrm{~d} x=\Gamma(n+1)=$ $n$ !. Thus, for real $z<1$, the symmetry relation for $\mathfrak{m}(t)$ gives:

$$
\begin{aligned}
\mathcal{M}(z) & =\int_{0}^{\infty} \mathfrak{m}(z t) e^{-t} \mathrm{~d} t=\int_{0}^{\infty} \mathfrak{m}(-z t) e^{-t(1-z)} \mathrm{d} t \\
& =\int_{0}^{\infty} \mathfrak{m}\left(t \frac{z}{z-1}\right) \frac{1}{(1-z)} e^{-t} \mathrm{~d} t=\mathcal{M}\left(\frac{z}{z-1}\right) \frac{1}{1-z} .
\end{aligned}
$$

Both integrals converge for $z<1$ (since $m_{L} \leq 1,|m(z)| \leq e^{z}$ ), hence for these values of $z$ we have the above identity. The function $\mathcal{M}(z)$ was initially defined for $|z|<1$; nevertheless, the above identity gives a holomorphic continuation of $\mathcal{M}(z)$ to the half plane $\Re z<1 / 2$.

Lemma 2. The function $\mathcal{M}(z)$ can be analytically continued to the domain $\mathbb{C} \backslash \mathbb{R}_{x>1}$. 
Proof. In fact, $\mathfrak{m}(t)=\int_{0}^{\infty} \exp \left(\frac{x}{x+1} t\right) \mathrm{d} F(x)$. As noted above, $|\mathfrak{m}(t)| \leq e^{t}$ for positive $t$ (actually, Lemma 1 combined with Proposition 3 gives a slightly better estimate). Therefore, for real $z<1$ we have:

$$
\mathcal{M}(z)=\int_{0}^{\infty} \int_{0}^{\infty} \exp \left(\frac{x}{x+1} z t\right) e^{-t} \mathrm{~d} F(x) \mathrm{d} t=\int_{0}^{\infty} \frac{1}{1-\frac{x}{x+1} z} \mathrm{~d} F(x) .
$$

Thus, $\mathcal{M}(z)$ is a certain Stieltjes transform of the distribution $F(x)$, and the statement of the lemma is a well-known (and almost obvious) fact about such transforms.

The system (6) gives us the expression of $M_{L}$ in terms of $m_{s}$. In fact, there exists one more system which is independent of the distribution $F(x)$; it simply encodes the relation among functions $t^{L}$ and $\left(\frac{t}{t+1}\right)^{S}$, given by

$$
t^{L}=\sum_{s \geq L}\left(\begin{array}{l}
s-1 \\
L-1
\end{array}\right)\left(\frac{t}{t+1}\right)^{s} \quad L \geq 1, \quad t \geq 0 .
$$

(This is the Taylor series for $\left(\frac{x}{1-x}\right)^{L}$, after an additional substitution $x=\frac{t}{t+1}$ ). Ultimately,

$$
M_{L}=\sum_{s \geq L}\left(\begin{array}{c}
s-1 \\
L-1
\end{array}\right) m_{s}
$$

For the convenience, we introduce a function

$$
G(z)=\frac{\mathcal{M}(z)-1}{z}=\sum_{L=1}^{\infty} m_{L} z^{L-1}=\int_{0}^{\infty} \frac{\frac{x}{x+1}}{1-\frac{x}{x+1} z} \mathrm{~d} F(x)=2 \int_{0}^{1} \frac{x}{1-x z} \mathrm{~d} F(x) .
$$

Next theorem is our main result about $G(z)$. The power series converges in the disc $|z| \leq 1$ (including the boundary, as can be inherited from (10); moreover, this implies that there exist all left derivatives of $G(z)$ at $z=1$ ). The integral converges in the cut plane $\mathbb{C} \backslash(1, \infty)$.

THEOREM 1. Let $m_{L}=\int_{0}^{\infty}\left(\frac{x}{x+1}\right)^{L} \mathrm{~d} F(x)$. Then the generating power function, defined as $G(z)=\sum_{L=1}^{\infty} m_{L} z^{L-1}$, has an analytic continuation to the domain $\mathbb{C} \backslash \mathbb{R}_{x>1}$. It satisfies the functional equation

$$
-\frac{1}{1-z}-\frac{1}{(1-z)^{2}} G\left(\frac{1}{1-z}\right)+2 G(z+1)=G(z)
$$

and also the symmetry property

$$
G(z+1)=-\frac{1}{z^{2}} G\left(\frac{1}{z}+1\right)-\frac{1}{z} .
$$

Moreover, $G(z)=o(1)$ as $z \rightarrow \infty$ and the distance from $z$ to $\mathbb{R}_{+}$tends to infinity.

Proof. In analogy to $\mathcal{M}(z)$, for real $z<0$ define the following function: $\mathcal{M}_{0}(z)=$ $\int_{0}^{\infty} M(z t) e^{-t} \mathrm{~d} t$. In view of (7), this integral converges for real $z<0$. Thus,

$$
\mathcal{M}_{0}(z)=\int_{0}^{\infty} \int_{0}^{\infty} \exp (x z t) e^{-t} \mathrm{~d} F(x) \mathrm{d} t=\int_{0}^{\infty} \frac{1}{1-x z} \mathrm{~d} F(x) .
$$


From argument akin to the one used in proving Lemma 2 we deduce that $\mathcal{M}_{0}(z)$ extends as an analytic function to the region $\mathbb{C} \backslash \mathbb{R}_{>0}$. In this domain we see that

$$
\frac{\mathcal{M}_{0}(z)-1}{z}=\frac{\mathcal{M}(z+1)-1}{z+1},
$$

which is the consequence of the algebraic identity

$$
\left(\frac{1}{1-x z}-1\right) \cdot \frac{1}{z}=\left(\frac{1}{1-\frac{x}{x+1}(z+1)}-1\right) \cdot \frac{1}{z+1} .
$$

The relation (13) is independent of the specific distribution function, it simply encodes the information contained in (10) about the relation of powers of $x$ to powers of $x /(x+1)$. On the other hand, the specific information about $F(x)$ is encoded in (6) or (7). The comparison of these two relations gives the desired functional equation for $G(z)$. In fact, for real $t<0$ the following estimate follows from (7) and Lemma 1: $|M(t)|=\left|\mathfrak{m}(t)\left(2-e^{t}\right)^{-1}\right| \leq|\mathfrak{m}(t)| \ll 1$; and thus for real $z<0$ we have:

$$
\begin{aligned}
\mathcal{M}(z) & =\int_{0}^{\infty} \mathfrak{m}(z t) e^{-t} \mathrm{~d} t=\int_{0}^{\infty}\left(2-e^{z t}\right) M(z t) e^{-t} \mathrm{~d} t \\
& =2 \mathcal{M}_{0}(z)-\int_{0}^{\infty} M(z t) e^{-t(1-z)} \mathrm{d} t=2 \mathcal{M}_{0}(z)-\mathcal{M}_{0}\left(\frac{z}{1-z}\right) \frac{1}{1-z} .
\end{aligned}
$$

Finally, the substitution (13) gives us the functional equation

$$
\frac{1-z}{1+z}-\frac{z}{1-z} \mathcal{M}\left(\frac{1}{1-z}\right)+2 \frac{z}{z+1} \mathcal{M}(z+1)=\mathcal{M}(z) .
$$

The principle of analytic continuation implies that this equation should be satisfied for all values of arguments in the region of holomorphicity of $\mathcal{M}(z)$. Direct inspection shows that for $G(z)=\frac{\mathcal{M}(z)-1}{z}$ this equation reads as (12). Also, the symmetry property is a reformulation of (9). This proves the first part of the Theorem.

Obviously, the last assertion follows from the integral representation of $G(z)$ given by (11).

We call $G(z)$ the dyadic period function, since its functional equation is completely parallel to a three term functional equations which are satisfied by rational period functions and period functions associated with Maass wave forms [28]. The word 'dyadic' refers to the binary origin of the distribution function $F(x)$. Indeed, thorough inspection shows that the multiplier 2 in equations (20) and (12) emerges exactly from the fact that every generation of $\mathcal{T}$ has twice as many members as a previous generation.

4. Uniqueness of $G(z)$. In this section, we prove the uniqueness of a function having the properties described in Theorem 1. Note that two functional equations for $G(z)$ can be merged into a single one. It is easy to check that

$$
\frac{1}{z}+\frac{1}{z^{2}} G\left(\frac{1}{z}\right)+2 G(z+1)=G(z)
$$


is equivalent to both together. In fact, the change $z \mapsto 1 / z$ in the equation (14) gives the symmetry property, and application of it to the term $G(1 / z)$ of the above recovers the functional equation (12).

Proposition 4. The function which satisfies the conditions of Theorem 1 is unique.

Proof. Suppose there exist two such functions. Then their difference $G_{0}(z)$ has the same behaviour at infinity, and it satisfies the homogenic form of the equation (14). Let $M=\sup _{-1 \leq x \leq 0}\left|G_{0}(x)\right|=\left|G_{0}\left(x_{0}\right)\right|, x_{0} \in[-1,0]$. We will show that $M=0$; by the principle of analytic continuation this will imply that $G_{0}(z) \equiv 0$. Let $z$ be real, $-1 \leq z \leq 0$. Let us substitute $z \mapsto z-n$ in the equation (14), $n \in \mathbb{N}, n \geq 1$, and divide it by $2^{n}$. Thus, we obtain:

$$
\frac{G_{0}(z-n)}{2^{n}}-\frac{G_{0}(z-n+1)}{2^{n-1}}=\frac{1}{2^{n}(z-n)^{2}} G_{0}\left(\frac{1}{z-n}\right) .
$$

Note that for $z$ in the interval $[-1,0], \frac{1}{z-n}$ belongs to the same interval as well. Now sum this over $n \geq 1$. The series on both sides are absolutely convergent, minding the behaviour of $G_{0}(z)$ at infinity. Therefore,

$$
-G_{0}(z)=\sum_{n=1}^{\infty} \frac{1}{2^{n}(z-n)^{2}} G_{0}\left(\frac{1}{z-n}\right) .
$$

The evaluation of the right hand side gives:

$$
\left|G_{0}(z)\right| \leq \sum_{n=1}^{\infty} \frac{1}{2^{n} n^{2}} M=\left(\frac{\pi^{2}}{12}-\frac{1}{2} \log ^{2} 2\right) M \quad \text { for }-1 \leq z \leq 0 .
$$

The constant is $<1$. Thus, unless $M=0$, this is contradictory for $z=x_{0}$. This proves the proposition.

Note the similarity between (15) and the expression for the Gauss-KuzminWirsing operator $\mathbf{W}$. The latter is defined for bounded smooth functions $f:[0,1] \rightarrow \mathbb{R}$ by the formula

$$
[\mathbf{W} f](x)=\sum_{k=1}^{\infty} \frac{1}{(k+x)^{2}} f\left(\frac{1}{k+x}\right)
$$

The eigenvalue 1 corresponds to the function $\frac{1}{1+x}$ (see [20] chapter III, for Kuzmin's treatment). The second largest eigenvalue $-0.303663 \ldots$ (the Wirsing constant) leads to a function with unknown analytic expression [44]; this eigenvalue determines the speed of convergence of iterates $\left[\mathbf{W}^{(n)} f\right](x)$ to $\frac{c}{1+x}$ (for certain $c \in \mathbb{R}$ ). The spectral analysis of our operator is presented in Section 6. Ref. [2] contains much more details and results in this direction.

Let $\Im z>0$. We remind that the Eisenstein series of weight 2 for $\mathrm{PSL}_{2}(\mathbb{Z})$ is defined as [39]

$$
G_{2}(z)=\sum_{n \in \mathbb{Z}} \sum_{m \in \mathbb{Z}}^{\prime} \frac{1}{(m+n z)^{2}}
$$


(mind the order of summation, since the series is not absolutely convergent). This series has the following Fourier expansion: if $q=e^{2 \pi i z}$, then

$$
G_{2}(z)=\frac{\pi^{2}}{3}-8 \pi^{2} \sum_{n=1}^{\infty} \sigma_{1}(n) q^{n}
$$

where $\sigma_{1}(n)=\sum_{d \mid n} d$. Then this function is not completely modular, but we have the following identities ([39], chapter VII):

$$
G_{2}(z+1)=G_{2}(z), \quad G_{2}(-1 / z)=z^{2} G_{2}(z)-2 \pi i z .
$$

Note that for $\Im z>0$, all arguments in (12) simultaneously belong to the upper half plane. It is surprising (but not coincidental) that the function $\frac{i}{2 \pi} G_{2}(z)$ satisfies the functional equation (12) for $\Im z>0$ (see the remarks in Section 8 about possible connections in idelic setting). To check this statement, note that

$\frac{i}{2 \pi} G_{2}\left(-\frac{1}{z-1}\right)=\frac{i}{2 \pi}\left((z-1)^{2} G_{2}(z-1)-2 \pi i(z-1)\right)=\frac{i}{2 \pi}(1-z)^{2} G_{2}(z)-(1-z)$.

Thus, plugging this into (12), we obtain an identity. If we define $G_{2}(z)=\overline{G_{2}(\bar{z})}$ for $\Im z<0$, one checks directly that the symmetry property is also satisfied. This is a surprising phenomena. See the last section of [2] for more speculations on this topic, where the space of dyadic period functions in the upper half plane (denoted by DPF ${ }^{0}$ ) is introduced.

We end this section with presenting a system of linear equations satisfied by the moments $m_{L}$. This system is derived from the three term functional equation (14) and is a superior result in numerical calculations: whereas directly from the definition we can recover only a few digits of the moments, this method allows to calculate up to 60 digits and more.

Proposition 5. Denote $c_{L}=\sum_{n=1}^{\infty} \frac{1}{2^{n} n^{L}}=L i_{L}\left(\frac{1}{2}\right)$. The moments $m_{s}$ satisfy the infinite system of linear equations

$$
m_{s}=\sum_{L=0}^{\infty}(-1)^{L} c_{L+s}\left(\begin{array}{c}
L+s-1 \\
s-1
\end{array}\right) m_{L}, \quad s \geq 1
$$

Proof. Indeed, for $\Re z \leq 0$ we have (recall that $m_{0}=1$ ):

$$
-G(z)=\sum_{n=1}^{\infty} \frac{1}{2^{n}(z-n)}+\sum_{n=1}^{\infty} \frac{1}{2^{n}(z-n)^{2}} G\left(\frac{1}{z-n}\right)=\sum_{n=1}^{\infty} \frac{1}{2^{n}} \sum_{L=0}^{\infty} m_{L}\left(\frac{1}{z-n}\right)^{L+1} .
$$

This series is absolutely and uniformly convergent for $\Re z \leq 0$, as is implied by (10). We obtain the needed result after taking the $s$ th left derivative at $z=0$.

Numerical calculations are presented in [3]. This method gives high-precision values for other constants, including the Kinney's constant.

5. Exponential generating function $\mathfrak{m}(t)$. The aim of this section is to interpret (14) in terms of $\mathfrak{m}(t)$. The following theorem, along with the boundary condition 
$\mathfrak{m}(0)=1$ and regularity property as in Lemma 1 , uniquely determines the function $\mathfrak{m}(t)$.

THEOREM 2. The function $\mathfrak{m}(s)$ satisfies the integral equation

$$
\mathfrak{m}(-s)=\left(2 e^{s}-1\right) \int_{0}^{\infty} \mathfrak{m}^{\prime}(-t) J_{0}(2 \sqrt{s t}) \mathrm{d} t, \quad s \in \mathbb{R}_{+},
$$

where $J_{0}(*)$ stands for the Bessel function: $J_{0}(z)=\frac{1}{\pi} \int_{0}^{\pi} \cos (z \sin x) \mathrm{d} x$.

Proof. For $\Re z<1$, we have that $G(z)=\int_{0}^{\infty} \mathfrak{m}^{\prime}(z t) e^{-t} \mathrm{~d} t$. Thus,

$G(z)=-\frac{1}{z} \int_{0}^{\infty} \mathfrak{m}^{\prime}(-t) e^{t / z} \mathrm{~d} t$ for $\Re z<0, \quad G(z)=\frac{1}{z} \int_{0}^{\infty} \mathfrak{m}^{\prime}(t) e^{-t / z} \mathrm{~d} t$ for $0<\Re z<1$.

Thus, the functional equation for $G(z)$ in the region $\Re z<-1$ in terms of $\mathfrak{m}^{\prime}(t)$ reads as

$$
\frac{1}{z}=\int_{0}^{\infty} \mathfrak{m}^{\prime}(-t)\left(\frac{2}{z+1} e^{\frac{t}{z+1}}+\frac{1}{z} e^{t z}-\frac{1}{z} e^{\frac{t}{z}}\right) \mathrm{d} t .
$$

Now, multiply this by $e^{-s z}$ and integrate over $\Re z=-\sigma<-1$, where $s>0$ is real. We have ([26], p. 465)

$$
\begin{aligned}
\int_{-\sigma-i \infty}^{-\sigma+i \infty} \frac{e^{-s z}}{z} \mathrm{~d} z & =-2 \pi i \\
2 \int_{-\sigma-i \infty}^{-\sigma+i \infty} \frac{e^{\frac{t}{z+1}-s z}}{z+1} \mathrm{~d} z & =-2 e^{s} \int_{\sigma-1-i \infty}^{\sigma-1+i \infty} \frac{e^{s z-\frac{t}{z}}}{z} \mathrm{~d} z \\
& =-2 e^{s} \int_{\sigma_{0}-i \infty}^{\sigma_{0}+i \infty} \frac{e^{\sqrt{s t}-\frac{\sqrt{s t}}{z}}}{z} \mathrm{~d} z=-4 \pi i e^{s} J_{0}(2 \sqrt{s t}),
\end{aligned}
$$

where $\sigma_{0}=(\sigma-1) \sqrt{\frac{t}{s}}>0$, and $J_{v}(*)$ stands for the Bessel function (see [26], p. 597 for the representation of the Bessel function by this integral). Further,

$$
\int_{-\sigma-i \infty}^{-\sigma+i \infty} \frac{e^{(t-s) z}}{z} \mathrm{~d} z=\left\{\begin{array}{ll}
-2 \pi i & \text { if } s>t, \\
0 & \text { if } s<t,
\end{array} \int_{-\sigma-i \infty}^{-\sigma+i \infty} \frac{e^{\frac{t}{z}-s z}}{z} \mathrm{~d} z=-2 \pi i J_{0}(2 \sqrt{s t}) .\right.
$$

Thus, eventually

$$
-2 \pi i=-2 \pi i \int_{0}^{\infty} \mathfrak{m}^{\prime}(-t)\left(2 e^{s}-1\right) J_{0}(2 \sqrt{s t}) \mathrm{d} t-2 \pi i \int_{0}^{s} \mathfrak{m}^{\prime}(-t) \mathrm{d} t
$$

since $\mathfrak{m}(0)=1$, this proves the proposition.

Thus, we have obtained an integral equation for $\mathfrak{m}(s)$, which corresponds to the functional equation (14) for $G(z)$. They are both, in fact, mere reformulations of (1) in different terms.

6. Dyadic eigenfunctions. In this Section, we introduce the sequence of functions $G_{\lambda}(z)$, which satisfy the functional equation analogous to (14). 
Since $J_{0}^{\prime}(*)=-J_{1}(*), J_{1}(0)=0$, integration by parts in (16) leads to

$$
\int_{0}^{\infty} \frac{\mathfrak{m}(-t)}{\sqrt{t}} J_{1}(2 \sqrt{s t}) \mathrm{d} t=\frac{1}{\sqrt{s}}-\frac{\mathfrak{m}(-s)}{\sqrt{s}\left(2 e^{s}-1\right)} .
$$

Recall that the Hankel transform of degree $v>-1 / 2$ of the function $f(r)$ (provided that $\int_{0}^{\infty} f(r) \sqrt{r} \mathrm{~d} r$ converges absolutely) is defined as

$$
g(\rho)=\int_{0}^{\infty} f(r) J_{v}(r \rho) r \mathrm{~d} r
$$

where $J_{v}(*)$ stands for the $v$ th Bessel function. The inverse is given by the Hankel inversion formula with exactly the same kernel ([43], chapter XIV, section 14.4.). Thus, after a proper change of variables, Hankel transform can be read as

$$
g(\rho)=\int_{0}^{\infty} f(r) J_{\nu}(2 \sqrt{r \rho}) \mathrm{d} r \Leftrightarrow f(r)=\int_{0}^{\infty} g(\rho) J_{\nu}(2 \sqrt{r \rho}) \mathrm{d} \rho .
$$

Thus, application of this inversion to the identity (18) yields

$$
\frac{\mathfrak{m}(-s)}{\sqrt{s}}=\int_{0}^{\infty} \frac{J_{1}(2 \sqrt{s t})}{\sqrt{t}} \mathrm{~d} t-\int_{0}^{\infty} \frac{\mathfrak{m}(-t)}{\sqrt{t}\left(2 e^{t}-1\right)} J_{1}(2 \sqrt{s t}) \mathrm{d} t .
$$

The first integral on the r.h.s. is equal $\mathrm{t}-\left.\frac{1}{\sqrt{s}} J_{0}(2 \sqrt{s t})\right|_{t=0} ^{\infty}=\frac{1}{\sqrt{s}}$. Let $\psi(s)=\left(2 e^{s}-1\right)^{1 / 2}$. Then this equation can be rewritten as

$$
\frac{\mathfrak{m}(-s)}{\sqrt{s} \psi(s)}=\frac{1}{\sqrt{s} \psi(s)}-\int_{0}^{\infty} \frac{\mathfrak{m}(-t)}{\sqrt{t} \psi(t)} \cdot \frac{J_{1}(2 \sqrt{s t})}{\psi(s) \psi(t)} \mathrm{d} t .
$$

Hence, if we denote

$$
\frac{J_{1}(2 \sqrt{s t})}{\psi(s) \psi(t)}=K(s, t), \quad \frac{\mathfrak{m}(-s)-1}{\sqrt{s} \psi(s)}=\mathbf{m}(s),
$$

we obtain a second type Fredholm integral equation with symmetric kernel ([22], Chapter 9):

$$
\begin{gathered}
\mathbf{m}(s)=\ell(s)-\int_{0}^{\infty} \mathbf{m}(t) K(s, t) \mathrm{d} t \\
\text { where } \ell(s)=-\frac{1}{\psi(s)} \int_{0}^{\infty} \frac{J_{1}(2 \sqrt{s t})}{\sqrt{t}\left(2 e^{t}-1\right)} \mathrm{d} t=\frac{1}{\sqrt{s} \psi(s)}\left(\sum_{n=1}^{\infty} e^{-s / n} 2^{-n}-1\right) .
\end{gathered}
$$

The behaviour at infinity of the Bessel function is given by an asymptotic formula

$$
J_{1}(x) \sim\left(\frac{2}{\pi x}\right)^{1 / 2} \cos \left(x-\frac{3}{4} \pi\right)
$$

([43], chapter VII, section 7.1). Therefore, obviously,

$$
\int_{0}^{\infty} \int_{0}^{\infty}|K(s, t)|^{2} \mathrm{~d} s \mathrm{~d} t<\infty, \quad \int_{0}^{\infty}|\ell(s)|^{2} \mathrm{~d} s<\infty .
$$


Thus, the operator associated with the kernel $K(s, t)$ is the Hilbert-Schmidt operator ([22], p. 532). The theorem of Hilbert-Schmidt ([22], p. 283) states that the solution of this type of integral equations reduces to finding the eigenvalues $\lambda$ and the eigenfunctions $A_{\lambda}(s)$. We postpone the solution of this integral equation for the future. Till the end of this section, we deal only with eigenfunctions. The integral operator, consequently, is a compact self-conjugate operator in the Hilbert space, it possesses a complete orthogonal system of eigenfunctions $A_{\lambda}(s)$, all $\lambda$ are real and $\lambda_{n} \rightarrow 0$, as $n \rightarrow \infty$. If we denote $A_{\lambda}(s) \psi(s)=B_{\lambda}(s)$, then the equation for an eigenfunction reads as

$$
\int_{0}^{\infty} B_{\lambda}(t) \frac{J_{1}(2 \sqrt{s t})}{2 e^{t}-1} \mathrm{~d} t=\lambda B_{\lambda}(s)
$$

This gives $B_{\lambda}(0)=0$. Since $A_{\lambda}(s) \in L_{2}(0, \infty)$, and $J_{1}(*)$ is bounded, this implies that $B_{\lambda}(s)$ is uniformly bounded for $s \geq 0$ as well. Moreover, since the Taylor expansion of $J_{1}(*)$ contains only odd powers of the variable, $B_{\lambda}(s) \sqrt{s}$ has a Taylor expansion with centre 0 and is an entire function. Now, multiply this by $\sqrt{s} e^{-s / z}, z>0$, and integrate over $s \in \mathbb{R}_{+}$. The Laplace transform of $\sqrt{s} J_{1}(2 \sqrt{s})$ is $\frac{1}{z^{2}} e^{-1 / z}$ ([26], p. 503). Thus, we obtain

$$
\frac{1}{\lambda} \int_{0}^{\infty} \frac{B_{\lambda}(t) \sqrt{t}}{2 e^{t}-1} e^{-t z} \mathrm{~d} t=\frac{1}{z^{2}} \int_{0}^{\infty} B_{\lambda}(s) \sqrt{s} e^{-\frac{s}{z}} \mathrm{~d} s
$$

Denote by $G_{\lambda}(-z)$ the function on both sides of this equality. Thus, $G_{\lambda}(z)$ is defined at least for $\Re z \leq 0$. Since $2 e^{t(z+1)}-e^{t z}=\left(2 e^{t}-1\right) e^{t z}$, we have

$$
\lambda\left(2 G_{\lambda}(z+1)-G_{\lambda}(z)\right)=\int_{0}^{\infty} B_{\lambda}(t) \sqrt{t} e^{t z} \mathrm{~d} t=\frac{1}{z^{2}} G_{\lambda}(1 / z) .
$$

Therefore, we have proved the first part of the following theorem.

THEOREM 3. For every eigenvalue $\lambda$ of the integral operator, associated with the kernel $K(s, t)$, there exists at least one holomorphic function $G_{\lambda}(z)$ (defined for $z \in \mathbb{C} \backslash \mathbb{R}_{>1}$ ), such that the following holds:

$$
2 G_{\lambda}(z+1)=G_{\lambda}(z)+\frac{1}{\lambda z^{2}} G_{\lambda}\left(\frac{1}{z}\right) .
$$

Moreover, $G_{\lambda}(z)$ for $\Re z<0$ satisfies all regularity conditions imposed by it being an image under the Laplace transform ([26], p. 469).

Conversely: for every $\lambda$, such that there exists a non-zero function, which satisfies (20) and these conditions, $\lambda$ is the eigenvalue of this operator. The set of all possible $\lambda s$ is countable, and $\lambda_{n} \rightarrow 0$, as $n \rightarrow \infty$.

Proof. The converse is straightforward, since, by the requirement, $G_{\lambda}(z)$ for $\Re z \leq 0$ is a Laplace image of a certain function, and all the above transformations are invertible. We leave the details. If the eigenvalue has multiplicity higher than 1 , then these $\lambda$-forms span a finite dimensional $\mathbb{C}$-vector space. Note that the proof of Proposition 1 implies $|\lambda|<0.342014_{+}$. Finally, the functional equation (20) gives the analytic continuation of $G_{\lambda}(z)$ to the half-plane $\Re z \leq 1$. Further, if $z \in \mathcal{U}$, where $\mathcal{U}=\{0 \leq \Re z \leq 1\} \backslash\{|z|<1\}$, we can continue $G_{\lambda}(z)$ to the region $\mathcal{U}+1$, and, inductively, to $\mathcal{U}+n, n \in \mathbb{N}$. Let $\mathcal{U}_{0}$ be 
the union of these. We can, obviously, continue $G_{\lambda}(z)$ to the set $\mathcal{U}_{0}^{-1}+n, n \in \mathbb{N}$. Similar iterations cover the described domain.

Note that, in contrast to $G(z)$, we do not have a symmetry property for $G_{\lambda}(z)$.

The following calculations produce the first few eigenvalues. Let the Taylor expansion of $G_{\lambda}(z)$ be given by

$$
G_{\lambda}(z)=\sum_{L=1}^{\infty} m_{L}^{(\lambda)} z^{L-1} .
$$

It converges in the unit circle, including its boundary (as is clear from (20), there exist all left derivatives at $z=1$ ). Thus, $m_{L}^{(\lambda)}$ have the same vanishing properties as $m_{L}$ (which guarantees the convergence of the series in (10)). And therefore, as in Proposition 5, we obtain:

$$
\lambda m_{s}^{(\lambda)}=\sum_{L=1}^{\infty}(-1)^{L-1} c_{L+s}\left(\begin{array}{c}
L+s-1 \\
s-1
\end{array}\right) m_{L}^{(\lambda)}, \quad s \geq 1 .
$$

Here $c_{L}=\sum_{n=1}^{\infty} \frac{1}{2^{n} n^{L}}$. If we denote $e_{s, L}=(-1)^{L-1} c_{L+s}\left(\begin{array}{c}L+s-1 \\ s-1\end{array}\right)$, then $\lambda$ is the eigenvalue of the infinite matrix $\mathcal{E}_{s, L=1}^{\infty}$. The numerical calculations with the augmentation of this matrix at sufficiently high level give the following first eigenvalues in decreasing order, with all digits exact:

$\lambda_{1}=0.25553210_{+}, \quad \lambda_{2}=-0.08892666_{+}, \quad \lambda_{3}=0.03261586_{+}, \quad \lambda_{4}=-0.01217621_{+}$.

Ref. [2] contains graphs of $G_{\lambda}(z)$ for the first six eigenvalues, and, more importantly, 'pair-correlation' results among different eigenvalues, including the 'eigenvalue' -1 , which corresponds precisely to $G(z)$. These results reveal the importance of $G_{\lambda}(z)$ in the study of $F(x)$.

7. $p$-adic distribution. In the previous sections, we were interested in the distribution of the $n$th generation of the tree $\mathcal{T}$ in the field of real numbers. Since the set of non-equivalent valuations of $\mathbb{Q}$ contains a valuation associated with any prime number $p$, it is natural to consider the distribution of the set of each generation in the field of $p$-adic numbers $\mathbb{Q}_{p}$. In this case we have an ultrametric inequality, which implies that two circles are either co-centric or do not intersect. We define

$$
F_{n}(z, v)=2^{-n+1} \#\left\{\frac{a}{b} \in \mathcal{T}^{(n)}: \operatorname{ord}_{p}\left(\frac{a}{b}-z\right) \geq v\right\}, \quad z \in \mathbb{Q}_{p}, \quad v \in \mathbb{Z} .
$$

(When $p$ is fixed, the subscript $p$ in $F_{n}$ is omitted). Note that in order to calculate $F_{n}(z, v)$ we can confine to the case $\operatorname{ord}_{p}(z)<v$; otherwise $\operatorname{ord}_{p}\left(\frac{a}{b}-z\right) \geq v \Leftrightarrow \operatorname{ord}_{p}\left(\frac{a}{b}\right) \geq$ $v$. We shall calculate the limit distribution $\mu_{p}(z, v)=\lim _{n \rightarrow \infty} F_{n}(z, v)$, and also some characteristics of it, e.g. the zeta function

$$
Z_{p}(s)=\int_{u \in \mathbb{Q}_{p}}|u|^{s} d \mu_{p}, \quad s \in \mathbb{C}, \quad z \in \mathbb{Q}_{p},
$$

where $|*|$ stands for the $p$-adic valuation. 
To illustrate how the method works, we will calculate the value of $F_{n}$ in two special cases. Let $p=2$ and let $E(n)$ be the number of rational numbers in the $n$th generation with one of $a$ or $b$ being even, and let $O(n)$ be the corresponding number fractions with both $a$ and $b$ odd. Then $E(n)+O(n)=2^{n-1}$. Since $\frac{a}{b}$ in the $n$th generation generates $\frac{a}{a+b}$ and $\frac{a+b}{b}$ in the $(n+1)$ st generation, each fraction $\frac{a}{b}$ with one of the $a, b$ even will generate one fraction with both numerator and denominator odd. If both $a, b$ are odd, then their two offsprings will not be of this kind. Therefore, $O(n+1)=E(n)$. Similarly, $E(n+1)=E(n)+2 O(n)$. This gives the recurrence $E(n+1)=E(n)+2 E(n-1), n \geq$ 2 , and this implies

$$
E(n)=\frac{2^{n}+2(-1)^{n}}{3}, \quad O(n)=\frac{2^{n-1}+2(-1)^{n-1}}{3}, \quad \mu_{2}(0,0)=\frac{2}{3} .
$$

(For the last equality note that $\frac{a}{b}$ and $\frac{b}{a}$ simultaneously belong to $\mathcal{T}^{(n)}$, and so the number of fractions with $\operatorname{ord}_{2}(*)>0$ is $\left.E(n) / 2\right)$. We will generalise this example to odd prime $p \geq 3$. Let $L_{i}(n)$ be the part of the fractions in the $n$th generation such that $a b^{-1} \equiv i \bmod p$ for $0 \leq i \leq p-1$ or $i=\infty$ (that is, $\left.b \equiv 0 \bmod p\right)$. Thus,

$$
\sum_{i \in \mathbb{F}_{p} \cup \infty} L_{i}(n)=1
$$

in other words, $L_{i}(n)=F_{n}(i, 1)$. For our later investigations we need a result from the theory of finite Markov chains.

Lemma 3. Let $\mathbf{A}$ be a matrix of a finite Markov chain with stages. That is, $a_{i, j} \geq 0$ and $\sum_{j=1}^{s} a_{i, j}=1$ for all $i$. Suppose that $\mathbf{A}$ is irreducible (for all pairs ( $i, j$ ), and some $m$, the entry $a_{i, j}^{(m)}$ of the matrix $\mathbf{A}^{m}$ is strictly positive), acyclic and recurrent (this is satisfied, if all entries of $\mathbf{A}^{m}$ are strictly positive for some $m$ ). Then the eigenvalue 1 is simple and if $\lambda$ is another eigenvalue, then $|\lambda|<1$, and $\mathbf{A}^{m}$, as $m \rightarrow \infty$, tends to the matrix $\mathbf{B}$, with entries $b_{i, j}=\pi_{j}$, where $\left(\pi_{1}, \ldots, \pi_{s}\right)$ is a unique left eigenvector with eigenvalue 1 , such that $\sum_{j=1}^{s} \pi_{j}=1$.

A proof of this lemma can be found in [17], Section 3.1., Theorem 1.3.

Proposition 6. $\mu_{p}(z, 1)=\frac{1}{p+1}$ for $z \in \mathbb{Z}_{p}$.

Proof. Similarly as in the above example, a fraction $\frac{a}{b}$ from the $n$th generation generates $\frac{a}{a+b}$ and $\frac{a+b}{b}$ in the $(n+1)$ st generation, and it is routine to check that

$$
L_{i}(n+1)=\frac{1}{2} L_{\frac{i}{1-i}}(n)+\frac{1}{2} L_{i-1}(n) \quad \text { for } \quad i \in \mathbb{F}_{p} \cup\{\infty\},
$$

(Here we make a natural convention for $\frac{i}{1-i}$ and $i-1$, if $i=1$ or $\infty$ ). In this equation, it can happen that $i-1 \equiv \frac{i}{1-i} \bmod p$; thus, $(2 i-1)^{2} \equiv-3 \bmod p$. The recurrence for this particular $i$ is to be understood in the obvious way, $L_{i}(n+1)=L_{i-1}(n)$. Therefore, if we denote the vector-column $\left(L_{\infty}(n), L_{0}(n), \ldots, L_{p-1}(n)\right)^{T}$ by $\mathbf{v}_{n}$, and if $\mathcal{A}$ is a matrix of the system (21), then $\mathbf{v}_{n+1}=\mathcal{A} \mathbf{v}_{n}$, and hence

$$
\mathbf{v}_{n}=\mathcal{A}^{n-1} \mathbf{v}_{1},
$$


where $\mathbf{v}_{1}=(0,0,1,0, \ldots, 0)^{T}$. In any particular case, this allows us to find the values of $L_{i}$ explicitly. For example, if $p=7$, the characteristic polynomial is

$$
f(x)=\frac{1}{16}(x-1)(2 x-1)\left(2 x^{2}+1\right)\left(4 x^{4}+2 x^{3}+2 x+1\right) .
$$

The list of roots is

$$
\alpha_{1}=1, \quad \alpha=\frac{1}{2}, \quad \alpha_{3,4}= \pm \frac{i}{\sqrt{2}}, \quad \alpha_{5,6,7,8}=\frac{-1-\sqrt{17}}{8} \pm \frac{\sqrt{1+\sqrt{17}}}{2 \sqrt{2}},
$$

(with respect to the two values for the root $\sqrt{17}$ ), the matrix is diagonalisible, and the Jordan normal form gives the expression

$$
L_{i}(n)=\sum_{s=1}^{8} C_{i, s} \alpha_{s}^{n}
$$

Note that the elements in each row of the $(p+1) \times(p+1)$ matrix $\mathcal{A}$ are non-negative and sum up to 1 , and thus, we have a matrix of a finite Markov chain. We need to check that it is acyclic. Let $\tau(i)=i-1$, and $\varsigma(i)=\frac{i}{1-i}$ for $i \in \mathbb{F}_{p} \cup\{\infty\}$. The entry $a_{i, j}^{(m)}$ of $\mathcal{A}^{m}$ is

$$
a_{i, j}^{(m)}=\sum_{i_{1}, \ldots, i_{m-1}} a_{i, i_{1}} \cdot a_{i_{1}, i_{2}} \cdot \ldots \cdot a_{i_{m-1}, j}
$$

Therefore, we need to check that for some fixed $m$, the composition of $m s^{\prime} s$ or $\tau^{\prime} s$ leads from any $i$ to any $j$. One checks directly that for any positive $k$, and $i, j \in \mathbb{F}_{p}$,

$$
\begin{aligned}
\tau^{p-1-j} \circ \varsigma \circ \tau^{k} & \circ \varsigma \circ \tau^{i-1}(i)=j, \\
\tau^{p-1-j} & \circ \varsigma \circ \tau^{k}(\infty)=j, \\
\tau^{k} & \circ \varsigma \circ \tau^{i-1}(i)=\infty ;
\end{aligned}
$$

(for $i=0$, we write $\tau^{-1}$ for $\tau^{p-1}$ ). For each pair $(i, j)$, choose $k$ in order the amount of compositions used to be equal (say, to $m$ ). Then obviously all entries of $\mathcal{A}^{m}$ are positive, ant this matrix satisfies the conditions of Lemma 3. Since all columns also sum up to $1,\left(\pi_{1}, \ldots, \pi_{p+1}\right), \pi_{j}=\frac{1}{p+1}, 1 \leq j \leq p+1$, is the needed eigenvector. This proves the proposition.

The next theorem describes $\mu(z, v)$ in all cases.

THEOREM 4. Let $v \in \mathbb{Z}$ and $z \in \mathbb{Q}_{p}$, and $\operatorname{ord}_{p}(z)<v($ or $z=0)$. Then, if $z$ is a $p$-adic integer,

$$
\mu(z, v)=\frac{1}{p^{v}+p^{v-1}} .
$$

If $z$ is not p-adic integer, $\operatorname{ord}_{p}(z)=-\lambda<0$,

$$
\mu(z, v)=\frac{1}{p^{v+2 \lambda}+p^{v+2 \lambda-1}} .
$$


For $z=0,-v \leq 0$, we have

$$
\mu(0,-v)=1-\frac{1}{p^{v+1}+p^{v}} .
$$

This theorem allows the computation of the associated zeta-function:

COROLlary 1. For $s$ in the strip $-1<\Re s<1$,

$$
Z_{p}(s)=\int_{u \in \mathbb{Q}_{p}}|u|^{s} d \mu_{p}=\frac{(p-1)^{2}}{\left(p-p^{-s}\right)\left(p-p^{s}\right)},
$$

and $Z_{p}(s)=Z_{p}(-s)$.

The proof is straightforward. It should be noted that this expression encodes all the values of $\mu(0, v)$ for $v \in \mathbb{Z}$.

Proof of Theorem 4. For shortness, when $p$ is fixed, denote $\operatorname{ord}_{p}(*)$ by $v(*)$. As before, we want a recurrence relation among the numbers $F_{n}(i, \kappa), i \in \mathbb{Q}_{+}$. For each $\kappa \in \mathbb{Z}$, we can confine to the case $i<p^{\kappa}$. If $i=0$, we only consider $\kappa>0$ and call these pairs $(i, \kappa)$ 'admissible'. In these recurrence relations we are forced to include also $G_{n}(0,-\kappa)$ for $\kappa \geq 1$, which are defined this way:

$$
G_{n}(0,-\kappa)=2^{-n+1} \#\left\{\frac{a}{b} \in \mathcal{T}^{(n)}: v\left(\frac{a}{b}\right) \leq-\kappa\right\}, \quad z \in \mathbb{Q}_{p}, \quad \kappa \in \mathbb{Z} .
$$

(note that compared with the definition of $F_{n}$, the inequality is reversed). As before, a fraction $\frac{a}{b}$ in the $n$th generation generates the fractions $\frac{a}{a+b}$ and $\frac{a+b}{b}$ in the $(n+1)$ st generation. Let $\tau(i, \kappa)=\left((i-1) \bmod p^{\kappa}, \kappa\right)$. Then for all admissible pairs $(i, \kappa), i \neq 0$, the pair $\tau(i, \kappa)$ is also admissible, and

$$
v\left(\frac{a+b}{b}-i\right)=\kappa \Leftrightarrow v\left(\frac{a}{b}-(i-1)\right)=\kappa .
$$

Second, if $\frac{a}{a+b}=i+p^{\kappa} u, i \neq 1, u \in \mathbb{Z}_{p}$, and $(i, \kappa)$ is admissible, then

$$
\frac{a}{b}-\frac{i}{1-i}=\frac{p^{\kappa} u}{(1-i)\left(1-i-p^{\kappa} u\right)} .
$$

Since $v\left(\frac{i}{1-i}\right)=v(i)-v(1-i)$, this is 0 unless $i$ is an integer, equals to $v(i)$ if the latter is $>0$ and equals to $-v(1-i)$ if $v(1-i)>0$. Further, this difference has valuation $\geq \kappa_{0}=\kappa$, if $i \in \mathbb{Z}, i \not \equiv 1 \bmod p$, valuation $\geq \kappa_{0}=\kappa-2 v(1-i)$, if $i \in \mathbb{Z}, i \equiv 1 \bmod p$, and valuation $\geq \kappa_{0}=\kappa-2 v(i)$ if $i$ is not integer. In all three cases, easy to check, that, if we define $i_{0}=\frac{i}{1-i} \bmod p^{\kappa_{0}}$, the pair $\varsigma(i, \kappa)={ }^{\operatorname{def}}\left(i_{0}, \kappa_{0}\right)$ is admissible. For the converse, let $\frac{a}{b}=i_{0}+p^{\kappa 0} u, u \in \mathbb{Z}_{p}$. Then

$$
\frac{a}{a+b}-\frac{i_{0}}{1+i_{0}}=\frac{p^{\kappa_{0}}}{\left(1+i_{0}+p^{\kappa_{0}} u\right)\left(1+i_{0}\right)} .
$$

If $i=\frac{i_{0}}{1+i_{0}}$ is a $p$-adic integer, $i \not \equiv 1 \bmod p$, this has a valuation $\geq \kappa=\kappa_{0}$; if $i$ is a $p$ adic integer, $i \equiv 1(p)$, this has valuation $\geq \kappa=\kappa_{0}-2 v\left(i_{0}\right)=\kappa_{0}+2 v(1-i)$; if $i$ is not 
a $p$-adic integer, this has valuation $\geq \kappa=\kappa_{0}-2 v\left(1+i_{0}\right)=\kappa_{0}+2 v(i)$. Thus,

$$
v\left(\frac{a}{a+b}-i\right) \geq \kappa \Leftrightarrow v\left(\frac{a}{b}-i_{0}\right) \geq \kappa_{0} .
$$

Let $i=1$. If $\frac{a}{a+b}=1+p^{\kappa} u$, then $\kappa>0, u \in \mathbb{Z}_{p}$, and we obtain $\frac{a}{b}=-1-\frac{1}{p^{\kappa} u}, v\left(\frac{a}{b}\right) \leq$ $-\kappa$. Converse is also true. Finally, for $\kappa \geq 1$,

$$
v\left(\frac{a+b}{b}\right) \leq-\kappa \Leftrightarrow v\left(\frac{a}{b}\right) \leq-\kappa,
$$

and

$$
v\left(\frac{a}{a+b}\right) \leq-\kappa \Leftrightarrow v\left(\frac{a}{b}+1\right) \geq \kappa .
$$

Therefore, we have the recurrence relations:

$$
\left\{\begin{array}{l}
F_{n+1}(i, \kappa)=\frac{1}{2} F_{n}(\tau(i, \kappa))+\frac{1}{2} F_{n}(\varsigma(i, \kappa)), \text { if }(i, \kappa) \text { is admissible } \\
F_{n+1}(1, \kappa)=\frac{1}{2} F_{n}(0, \kappa)+\frac{1}{2} G_{n}(0,-\kappa), \kappa \geq 1 \\
G_{n+1}(0,-\kappa)=\frac{1}{2} G_{n}(0,-\kappa)+\frac{1}{2} F_{n}(-1, \kappa), \kappa \geq 1
\end{array}\right.
$$

Thus, we have an infinite matrix $\mathcal{A}$, which is a change matrix for the Markov chain. If $\mathbf{v}_{n}$ is an infinite vector-column of $F_{n}^{\prime} \mathrm{s}$ and $G_{n}^{\prime} \mathrm{s}$, then $\mathbf{v}_{n+1}=\mathcal{A} \mathbf{v}_{n}$, and, as before, $\mathbf{v}_{n}=\mathcal{A}^{n-1} \mathbf{v}_{1}$. It is direct to check that each column also contains exactly two non-zero entries $\frac{1}{2}$, or one entry, equal to 1 . In terms of Markov chains, we need to determine the classes of orbits. Then in proper rearranging, the matrix $\mathcal{A}$ looks like

$$
\left(\begin{array}{ccccc}
\mathbf{P}_{1} & 0 & \ldots & 0 & \ldots \\
0 & \mathbf{P}_{2} & \ldots & 0 & \ldots \\
\vdots & & \ddots & \vdots & \vdots \\
0 & 0 & \ldots & \mathbf{P}_{s} & 0 \\
\vdots & \vdots & \ldots & 0 & \ddots
\end{array}\right)
$$

where $\mathbf{P}_{s}$ are finite Markov matrices. Thus, we claim that the length of each orbit is finite, every orbit has a representative $G_{*}(0,-\kappa), \kappa \geq 1$, the length of it is $p^{\kappa}+p^{\kappa-1}$, and the matrix is recurrent (that is, every two positions communicate). In fact, from the system above and form the expression of the maps $\tau(i, \kappa)$ and $\varsigma(i, \kappa)$, the direct check shows that the complete list of the orbit of $G_{*}(0,-\kappa)$ consists of (and each pair of states are communicating):

$$
\begin{gathered}
G_{*}(0,-\kappa), \\
F_{*}(i, \kappa) \quad\left(i=0,1,2, \ldots, p^{\kappa}-1\right), \\
F_{*}\left(p^{-\lambda} u, \kappa-2 \lambda\right) \quad\left(\lambda=1,2, \ldots, \kappa-1, u \in \mathbb{N}, u \neq 0 \bmod p, u \leq p^{\kappa-\lambda}\right) .
\end{gathered}
$$

In total, we have

$$
1+p^{\kappa}+\sum_{\lambda=1}^{\kappa-1}\left(p^{\kappa-\lambda}-p^{\kappa-\lambda-1}\right)=p^{\kappa}+p^{\kappa-1}
$$


members in the orbit. Thus, each $\mathbf{P}_{\kappa}$ in the matrix above is a finite dimensional $\ell_{\kappa} \times \ell_{\kappa}$ matrix, where $\ell_{\kappa}=p^{\kappa}+p^{\kappa-1}$. For $\kappa=1$, the matrix $\mathbf{P}_{1}$ is exactly the matrix of the system (21). As noted above, the vector column $(1,1, \ldots, 1)^{T}$ is the left eigenvector. As in the previous Proposition, it is straightforward to check that this matrix is irreducible and acyclic (that is, the entries of $\mathbf{P}_{\kappa}^{n}$ are strictly positive for sufficiently large $n$ ). In fact, since by our observation, each two members in the orbit communicate, and since we have a move $G_{*}(0,-\kappa) \rightarrow G_{*}(0,-\kappa)$, the proof of the last statement is immediate: there exists $n$ such that any position is reachable from another in exactly $n$ moves, and this can be achieved at the expense of the move just described. Therefore, all entries of $\mathbf{P}_{\kappa}^{n}$ are strictly positive. Thus, the claim of the Theorem follows from the Lemma 3.

8. Conclusion. We end the paper with the following remarks. As is implied by Theorem 4, the measure $\mu_{p}$ of those rationals in the Calkin-Wilf tree which are invertible elements of $\mathbb{Z}_{p}$ is equal to $\frac{p-1}{p+1}$. We follow the line of the Tate thesis [7], and modify this measure so that $\mathbb{Z}_{p}^{*}$ has measure 1 ; accordingly, let us define $\mu_{p}^{\prime}=\frac{p+1}{p-1} \mu_{p}$. Thus, we are lead to the formal definition of the zeta function

$$
\begin{aligned}
\zeta_{\mathcal{T}}(s) & =\prod_{p} \int_{u \in \mathbb{Q}_{p}}|u|^{s} \mathrm{~d} \mu_{p}^{\prime}=\prod_{p}\left(1-\frac{1}{p^{2}}\right) \prod_{p} \frac{1}{1-p^{-s-1}} \cdot \frac{1}{1-p^{s-1}} \\
& =\frac{6}{\pi^{2}} \zeta(s+1) \zeta(-s+1) .
\end{aligned}
$$

This product diverges everywhere; nevertheless, if we apply the functional equation of the Riemann $\zeta$ function for the second multiplier, we obtain

$$
\zeta_{\mathcal{T}}(s)=\frac{12}{\pi^{2}}(2 \pi)^{-s} \cos \left(\frac{\pi s}{2}\right) \Gamma(s) \zeta(s) \zeta(s+1) .
$$

From the above definition it is clear that, formally, this zeta function is the sum of the form $\sum_{r \in \mathbb{Q}^{+}} \mu_{r} r^{-s}$, where, if $r \in \mathbb{Q}_{+}$, and $\mu_{r}$ stands for the limit measure of those rationals in the $n$th generation of $\mathcal{T}$, which have precisely the same valuation as $r$ at every prime which appears in the decomposition of $r$, times the factor $\prod_{\operatorname{ord}_{p}(r) \neq 0} \frac{p+1}{p-1}$. Surprisingly, the product $\zeta(s) \zeta(s+1)$ is the zeta function of the Eisenstein series $G_{2}(z)$, which is related to the distribution of rationals in $\mathcal{T}$ at the infinite prime $\mathbb{Q}_{\infty}=\mathbb{R}$. In fact,

$$
\int_{0}^{\infty}\left(G_{2}(i z)-G_{2}(i \infty)\right) z^{s-1} \mathrm{~d} z=-8 \pi^{2}(2 \pi)^{-s} \Gamma(s) \zeta(s) \zeta(s+1) .
$$

This is a strong motivation to investigate the tree $\mathcal{T}$ and the Minkowski question mark function in a more general - idelic - setting, thus revealing the true connection between $p$-adic and real distribution, and clarifying the nature of continued fractions in this direction. We hope to realise this in subsequent papers.

Unfortunately, currently we left the most interesting question (the explicit description of the moments of ? $(x)$ ) unanswered. It is desirable to give the function $G(z)$ and dyadic forms $G_{\lambda}(z)$ certain other description than the one which arises directly from the tree $\mathcal{T}$. This is in part accomplished in [2] and [3]. These papers form a direct continuation of the current work. Among the other results, the dyadic zeta function $\zeta_{\mathcal{M}}(s)$ is introduced: it is given by $\zeta_{\mathcal{M}}(s) \Gamma(s+1)=\int_{0}^{\infty} x^{s} \mathrm{~d} F(x)$; the nature of dyadic 
eigenfunctions $G_{\lambda}(z)$ is clarified; certain integrals which involve $F(x)$ are computed; and finally, this research culminates with the proof that in the half plane $\Re z<1$ the dyadic period function $G(z)$ can be represented as an absolutely convergent series of rational functions with rational coefficients. Possibly, this technique can find its applications in the study of period functions for Maass wave forms.

ACKNOWLEDGEMENT. This research was partially supported by the Lithuanian State Science and Studies Foundation.

\section{REFERENCES}

1. G. Alkauskas, An asymptotic formula for the moments of Minkowski question mark function in the interval [0, 1], Lith. Math. J. 48(4) (2008), 357-367.

2. G. Alkauskas, Generating and zeta functions, structure, spectral and analytic properties of the moments of the Minkowski question mark function, Involve 2(2) (2009), 121-159.

3. G. Alkauskas, The Minkowski question mark function: Explicit series for the dyadic period function and moments, Math. Comp. Available at http://www.ams.org/mcom/0000000-00/S0025-5718-09-02263-7/home.html.

4. C. Bonanno, S. Graffi and S. Isola, Spectral analysis of transfer operators associated to Farey fractions, Atti Accad. Naz. Lincei Cl. Sci. Fis. Mat. Natur. Rend. Lincei (9) Mat. Appl. 19(1) (2008), 1-23.

5. C. Bonanno and S. Isola, Orderings of the rationals and dynamical systems, Colloq. Math. 116 (2009), 165-189. 363.

6. N. Calkin and H. Wilf, Recounting the rationals, Amer. Math. Mon. 107 (2000), 360

7. J. W. S. Cassels and A. Fröhlich (eds.), Algebraic number theory (Academic Press, London, 1967).

8. J. H. Conway, On numbers and games (A K Peters Ltd., Natick, MA, 2001) 82-86. $105-151$.

9. A. Denjoy, Sur une fonction réelle de Minkowski, J. Math. Pures Appl. 17 (1938),

10. K. Dilcher and K. B. Stolarsky, A polynomial analogue to the Stern sequence, Int. J. Number Theory 3(1) (2007), 85-103.

11. A. Dushistova and N. G. Moshchevitin, On the derivative of the Minkowski question mark funtion ?(x), arXiv:0706.2219.

12. M. D. Esposti, S. Isola and A. Knauf, Generalized Farey trees, transfer operators and phase transitions, Comm. Math. Phys. 275(2) (2007), 297-329.

13. S. R. Finch, Mathematical constants (Cambridge University Press, Cambridge, UK, 2003), 441-443, 151-154.

14. R. Girgensohn, Constructing singular functions via Farey fractions, J. Math. Anal. Appl. 203 (1996), 127-141.

15. P. J. Grabner, P. Kirschenhofer and R. F. Tichy, Combinatorial and arithmetical properties of linear numeration systems, Combinatorica 22(2) (2002), 245-267.

16. S. Isola, On the spectrum of Farey and Gauss maps, Nonlinearity 15 (2002), 1521-1539.

17. S. Karlin, A first course in stochastic processes (Academic Press, New York and London, 1968).

18. M. Kesseböhmer and B. O. Stratmann, A multifractal analysis for Stern-Brocot intervals, continued fractions and Diophantine growth rates, J. Reine Angew. Math. 605 (2007), 133-163.

19. M. Kesseböhmer and B. O. Stratmann, Fractal analysis for sets of non-differentiability of Minkowski's question mark function, J. Number Theory 128 (2008), 2663-2686.

20. A. Ya. Khinchin, Continued fractions (The University of Chicago Press, Chicago and London, 1964).

21. J. R. Kinney, Note on a singular function of Minkowski, Proc. Amer. Math. Soc. 11(5) (1960), 788-794.

22. A. N. Kolmogorov and S. V. Fomin, Elements of the theory of functions and functional analysis (Nauka, Moscow, 1989). Available at http://www.ams.org/mathscinetgetitem?mr=1025126. 
23. J. C. Lagarias, Number theory and dynamical systems, in The unreasonable effectiveness of number theory (Orono, ME, 1991), Amer. Math. Soc., Proc. Sympos. Appl. Math. 46 (1992), $35-72$.

24. J. C. Lagarias and C. P. Tresser, A walk along the branches of the extended Farey tree, IBM J. Res. Develop. 39(3) (1995), 788-794.

25. M. Lamberger, On a family of singular measures related to Minkowski's ? $(x)$ function, Indag. Math. N.S. 17(1) (2006), 45-63.

26. M. A. Lavrentjev and B. V. Shabat, Methods in the theory of functions of complex variable (Nauka, Moscow, 1987).

27. J. B. Lewis, Spaces of holomorphic functions equivalent to the even Maass cusp forms, Invent. Math. 127(2) (1997), 271-306.

28. J. B. Lewis and D. Zagier, Period functions for Maass wave forms. I, Ann. Math. (2), 153(1) (2001), 191-258.

29. H. Minkowski, Zur Geometrie der Zahlen, Verhandlungen des III Internationalen Mathematiker-Kongresous (Heidelberg 1904), 164-173. [Also: Werke, vol. II, 43-52.].

30. M. Newman, Recounting the rationals, Amer. Math. Mon. 110 (2003), 642-643.

31. H. Okamoto and M. Wunsch, A geometric construction of continuous, strictly increasing singular functions, Proc. Japan Acad. 83 Ser. A (2007), 114-118.

32. G. Panti, Multidimensional continued fractions and a Minkowski function, Monatsh. Math. 154(3) (2008), 247-264.

33. J. Paradís, P. Viader and L. Bibiloni, A new light on Minkowski's ?(x) function, $J$. Number Theory 73(2) (1998), 212-227.

34. J. Paradís, P. Viader and L. Bibiloni, The derivative of Minkowski's ?(x) function, $J$. Math. Anal. Appl. 253(1) (2001), 107-125.

35. G. Ramharter, On Minkowski's singular function, Proc. AMS 99(3), (1987), 596-597.

36. S. Reese, Some Fourier-Stieltjes coefficients revisited, Proc. Amer. Math. Soc. 105(2) (1989), 384-386.

37. F. Ryde, On the relation between two Minkowski functions, J. Number Theory 77 (1983), $47-51$.

38. R. Salem, On some singular monotonic functions which are strictly increasing, Trans. Amer. Math. Soc. 53(3) (1943), 427-439.

39. J.-P. Serre, A course in arithmetic, in Graduate texts in mathematics (Springer, New York and Heidelberg, 1996).

40. N. Sloane, The On-line Encyclopedia of integer sequences. Available at http://www. research.att.com/ $\sim$ njas/sequences/

41. M. A. Stern, Über eine zahlentheoretische Funktion, J. Reine Angew. Math. 55 (1858), $193-220$

42. R. F. Tichy, J. Uitz, An extension of Minkowski's singular function, Appl. Math. Lett. 8(5) (1995), 39-46.

43. G. N. Watson, A treatise on the theory of Bessel functions, 2nd ed. (Cambridge University Press, Cambridge, UK, 1996).

44. E. Wirsing, On the theorem of Gauss-Kuzmin-Lévy and a Frobenius-type theorem for function spaces, Acta Arith. 24 (1973/74), 507-528.

45. E. Wirsing, Jörn Steuding's Problem, Palanga 2006 (preprint).

46. D. Zagier, New points of view on the Selberg zeta function (preprint). 